\title{
6 Shared Decision-Making bei Kreuzschmerzen
}

Sabine Beck und Andreas Loh

\subsection{Kreuzschmerzen: häufig und teuer - aber unkompliziert?}

\section{Bedeutung der Kreuzschmerzen}

Unter Kreuzschmerzen versteht man Schmerzen oder Unwohlsein im Bereich des Rückens vom unteren Rippenbogen bis zur Glutäalfalte, die zu Einschränkungen bei den täglichen Verrichtungen führen. In Deutschland beträgt die Lebenszeitprävalenz für Kreuzschmerzen ca. 80\% (Schmidt et al. 2007), das heißt, dass jeder, der alt genug wird, irgendwann in seinem Leben sehr wahrscheinlich unter Kreuzschmerzen leidet. In Hausarztpraxen sind Kreuzschmerzen einer der häufigsten Anlässe, weswegen Patienten ihren Arzt aufsuchen (Praxisprävalenz/Woche 8\%) (Becker et al. 2003). Die durch Kreuzschmerzen verursachten Arbeitsunfähigkeitszeiten führen zum Ausfall von insgesamt $4 \%$ der gesamten Arbeitskraft in Deutschland (Göbel 2001). Damit sind sowohl die Relevanz im klinischen Alltag als auch die volkswirtschaftliche Bedeutung sehr hoch.

\section{Unkomplizierte und komplizierte Kreuzschmerzen}

Eine klare strukturelle Zuordnung von Kreuzschmerzen ist in den meisten Fällen nicht möglich. Zur adäquaten Behandlung ist weniger eine genaue ätiologische Ursachenklärung wichtig als vielmehr die Differenzierung zwischen komplizierten und unkomplizierten Kreuzschmerzen. Komplizierte Kreuzschmerzen können durch anamnestische Hinweise und Untersuchungs- 
befunde von unkomplizierten Kreuzschmerzen abgegrenzt werden. Wenn komplizierte Kreuzschmerzen vorliegen, muss weitere Diagnostik eingeleitet werden, um schwerwiegende Erkrankungen mit möglichem gefährlichen Verlauf auszuschließen. Zu den Warnhinweisen für komplizierte Kreuzschmerzen gehören z. B. sehr junges ( $<20$ Jahre) oder höheres ( $>50$ Jahre) Alter, schlechter Allgemeinzustand, Fieber, eine bekannte Tumorerkrankung oder Immunsuppression, ein vorausgegangenes Trauma oder bekannte Osteoporose sowie zunehmender Schmerz trotz Therapie und ausgeprägte neurologische Ausfälle. Für die weitere Behandlung ist hier die diagnostische Klärung und Zuordnung maßgebend. In diesem Kapitel soll daher im Weiteren lediglich die Behandlung von umkomplizierten Kreuzschmerzen betrachtet werden.

\section{Chronische Kreuzschmerzen - psychosoziale Risikofaktoren}

Die Prognose von unkomplizierten Kreuzschmerzen ist günstig, die meisten Beschwerden bessern sich innerhalb von 4-6 Wochen und die Mehrzahl der Patienten kann innerhalb dieser Zeit wieder an ihren Arbeitsplatz zurückkehren. Bei vielen Patienten treten Kreuzschmerzen gelegentlich wieder auf und haben erneut eine gute Prognose, bei einem Teil der Patienten jedoch chronifizieren die Schmerzen. Von chronischen Kreuzschmerzen spricht man bei Beschwerden von mehr als 12 Wochen Dauer. Durch langfristige Arbeitsunfähigkeitszeiten und Frühberentungen sind diese Patienten für die volkswirtschaftlichen Folgen von Kreuzschmerzen besonders relevant. Ebenso nehmen diese Patienten durch wiederholte Konsultationen, Krankschreibungen und Arznei- und Hilfsmittelverordnungen in der ärztlichen Versorgung einen besonderen Stellenwert ein.

Patienten mit chronischen Kreuzschmerzen unterscheiden sich von Patienten mit einem günstigen Verlauf weniger durch medizinische als vielmehr durch psychosoziale Faktoren. Ein geringer Bildungsstand, anhaltende Belastungen im privaten Alltag und Unzufriedenheit am Arbeitsplatz sind Risikofaktoren für einen chronischen Verlauf von Kreuzschmerzen (Biering-Soerensen und Thomsen 1986, Deyo und Diehl 1988, Lehmann et al. 1993).

\subsection{Arzt-Patient-Kommunikation bei Kreuzschmerzen - unbequeme Wahrheiten}

\section{Unspezifische Ätiologie}

Kreuzschmerzen können selten einer klaren anatomischen Struktur zugeordnet werden, bei $85 \%$ der Patienten gelingt keine eindeutige ursächliche Klärung der Schmerzen (White 1982). Zumeist kann dabei ein Zusammenwirken mehrerer anatomischer Strukturen angenommen werden, dabei spielt eine zusätzliche schmerzbedingte muskuläre Verspannung sicher häufig eine mitverursachende Rolle. 
So sind Ärzte und Patienten damit konfrontiert, dass sich eine der häufigsten Gesundheitsstörungen für beide Seiten nur unbefriedigend klären lässt. Das Laienverständnis von Kreuzschmerzen geht zumeist von einer klar zuordenbaren mechanischen Ursache von Kreuzschmerzen aus: ein „verrutschter“ Wirbel, eine „herausgehüpfte“ Bandscheibe, ein „verklemmter“ Nerv werden als Grund der Beschwerden vermutet. Patienten wünschen verständlicherweise eine Klärung der Beschwerden bevor sie sich auf eine Behandlung einlassen. Durch die eigene Vermutung einer mechanischen Ursache drängen Patienten daher häufig auf eine Bildgebung.

Eine bildgebende Untersuchung kann dabei unergiebig bleiben oder degenerative Veränderungen zeigen, die nur fraglich mit der aktuellen Symptomatik in kausalem Zusammenhang stehen und den Patienten im ungünstigsten Fall fälschlicherweise davon überzeugen, eine dauerhafte Schädigung der Wirbelsäule zu haben. Der Arzt sieht sich also vor der Aufgabe, dem Patienten sowohl die unspezifische Ätiologie als auch den fehlenden Benefit einer Bildgebung zu vermitteln.

\section{Therapieziel: Bewegung, Vermeidung von Bettruhe und Krankschreibung}

Das Ziel der Behandlung der unkomplizierten Kreuzschmerzen ist die rasche Wiederaufnahme der üblichen Bewegung im Alltag. Durch eine möglichst schnelle Rückkehr zur gewohnten Aktivität werden die Beschwerden schneller gelindert und das Risiko einer Chronifizierung und langfristigen Krankschreibung vermindert. Durch Bewegungsvermeidung aus Angst vor erneuten Schmerzen können die aktuellen Beschwerden verstärkt und verlängert werden.

Für einen schmerzgeplagten Patienten ist der Ratschlag, sich weiterhin möglichst wie gewohnt zu bewegen zunächst wenig attraktiv und einleuchtend. Die meisten Patienten werden den Schmerz als Warnsignal verstehen und daraus folgern, dass Ruhe und Schonung angezeigt sind und Besserung bringen werden. Diese Bewertung von Schmerzen ist im Alltag für die meisten gängigerweise auftretenden Schmerzen durchaus richtig und führt dabei normalerweise zu sinnvollem abwartenden Schonverhalten.

Bei der Behandlung von Kreuzschmerzen ist die Beratung und aktive Einbeziehung des Patienten wesentlich zum Erreichen des Therapieziels. Dabei muss sich der Arzt der Aufgabe stellen, dem Patienten einen zunächst unverständlichen Therapieansatz zu vermitteln. Das Erreichen der Therapieziele ist letztendlich von der Motivationslage des Patienten abhängig. Mit einer gemeinsam erreichten und verantworteten Therapieentscheidung wird sich der Patient mehr identifizieren und im eigenen Interesse eine größere Motivation aufbringen, um die angestrebten Verhaltensänderungen umzusetzen.

\section{Kommunikationsstrategien}

Ziel der Kommunikation zwischen Arzt und Patient im Rahmen einer Konsultation aufgrund von Kreuzschmerzen ist zum einen, dem Patienten die 
notwendigen Informationen zu Diagnostik und Therapie zu vermitteln und zum anderen ein tragfähiges Therapiebündnis aufzubauen, was zu einer gemeinsam verantworteten Therapieentscheidung führt. Dabei ist es sinnvoll, zunächst die Annahmen und eigenen Krankheitskonzepte des Patienten zu eruieren und ernst zu nehmen und die Widersprüche zu evidenzbasiertem medizinischen Wissen bewusst zu thematisieren. Der Patient wird sich gerade dann auf die notwendige Diskussion einlassen, wenn klar kommuniziert wird, dass, so er dies wünscht, eine gemeinsame Behandlungsentscheidung getroffen werden soll.

\subsection{Die gemeinsame Entscheidung - Handlungsschritte}

Um im Sinne des Shared Decision Making zu einer gemeinsam verantworteten Therapieentscheidung zu kommen, sind in der praktischen Umsetzung eine Reihe von Handlungsschritten nachzuvollziehen. Um eine gemeinsame Therapieentscheidung anzustoßen, wird der Arzt zunächst dem Patienten mitteilen, dass eine Entscheidung ansteht, zu der es grundsätzlich gleichwertige Behandlungsoptionen gibt und die von Arzt und Patient gleichberechtigt gemeinsam getroffen werden soll. Dann werden vom Arzt die Optionen mit ihren Vor- und Nachteilen erklärt und die Gedanken, Befürchtungen und Erwartungen des Patienten thematisiert. Danach können Präferenzen ausgesprochen und eine gemeinsame Entscheidung ausgehandelt werden (s. auch Handlungsschritte in der unterstehenden Box).

Bei allen Handlungsschritten muss der Arzt sich durch Rückfragen und Ermunterung zum Nachfragen vergewissern, dass der Patient die Erklärungen verstanden hat.

\section{Handlungsschritte zur Umsetzung des Shared Decision Making}

1. mitteilen, dass eine Entscheidung ansteht

2. Gleichberechtigung der Partner formulieren

3. über Wahlmöglichkeiten informieren („Equipoise")

4. Information über Vor- und Nachteile der Optionen geben

5. Verständnis, Gedanken und Erwartungen erfragen

6. Präferenzen ermitteln

7. Aushandeln

8. gemeinsame Entscheidung herbeiführen

9. Vereinbarung zur Umsetzung der Entscheidung treffen

Die anstehenden Handlungsschritte sollen im Weiteren anhand eines praktischen Fallbeispieles anschaulich erläutert werden: 
Ein 38-jähriger Bauarbeiter kommt in die Sprechstunde und berichtet über Kreuzschmerzen, die vor zwei Tagen beim Anheben einer schweren Steinplatte plötzlich aufgetreten sind. Die Schmerzen sind dumpf und bewegungsabhängig im Bereich der unteren LWS und strahlen nicht in die Beine aus. Neurologische Auffälligkeiten bestehen nicht. Am selben Tag habe er noch mit Mühe bis zum Feierabend weitergearbeitet, gestern habe er jedoch nach der Vesper die Arbeit einstellen müssen. Eine Tablette Ibuprofen 400, die ihm seine Frau gegeben habe, habe wenig an den Beschwerden geändert.

\section{Mitteilung der anstehenden gemeinsamen Entscheidung}

Das Angebot, die anstehende Entscheidung zwischen verschiedenen grundsätzlich gleichwertigen Behandlungsoptionen gleichberechtigt zu treffen, kann dem Patienten beispielhaft so mitgeteilt werden:

„Es gibt verschiedene Möglichkeiten, Ihre Beschwerden zu behandeln. Allein ausärztlicher Sicht kann ich nicht entscheiden, welche für Sie die beste sein wird.“

Dem Patient kann an dieser Stelle erläutert werden, dass das wichtigste Prinzip der Therapie von Kreuzschmerzen ist, rasch wieder zur gewohnten Bewegung zurückzukehren. Als Arzt können Sie ihm dazu erklären, welche Möglichkeiten es gibt, ihn dabei zu unterstützen und die Aktivität zu ermöglichen. Da der Patient jedoch selbst die Behandlung in seinem Alltag umsetzen muss, ist es aber sinnvoll, dass er selber mitentscheidet, welche Möglichkeit für ihn in Frage kommt.

\section{Vor- und Nachteile der Optionen}

Dem Patienten können nun die verschiedenen Therapieoptionen dargelegt werden. Bei Kreuzschmerzen gibt es die Möglichkeit verschiedener medikamentöser Therapieansätze (Paracetamol, NSAID, niedrigpotente Opioide, Muskelrelaxantien, pflanzliche Mittel), physiotherapeutische und physikalische Maßnahmen sowie die lokale Behandlung mit Einreibemitteln oder der Injektion von Lokalanästhetika. Der Patient muss Informationen dazu bekommen, welche Therapieformen nachgewiesenermaßen effektiv oder nicht effektiv sind und welche Vor- und Nachteile sich durch die Behandlung für ihn ergeben können. Beispielhaft soll das für einige Therapieoptionen anhand der folgenden Tabelle 14 gezeigt werden. 
VI Praxisbeispiele

Tab. 14 Beispielhafte Behandlungsoptionen mit Vor- und Nachteilen

\begin{tabular}{|c|c|c|}
\hline Behandlung & Vorteile & Nachteile \\
\hline Paracetamol & $\begin{array}{l}\text { gut verträglich, nachweislich } \\
\text { effektiv, durch Schmerzlinderung } \\
\text { erleichterte Aktivität }\end{array}$ & $\begin{array}{l}\text { selten Nebenwirkungen } \\
\text { Medikamenteneinnahme }\end{array}$ \\
\hline $\begin{array}{l}\text { Muskelentspannende } \\
\text { Medikamente }\end{array}$ & $\begin{array}{l}\text { durch Entspannung der Muskulatur } \\
\text { Schmerzlinderung und erleichterte } \\
\text { Aktivität, } \\
\text { nachweislich effektiv }\end{array}$ & $\begin{array}{l}\text { häufig Nebenwirkungen } \\
\text { bei längerer Einnahme Gefahr der } \\
\text { Abhängigkeit }\end{array}$ \\
\hline Krankengymnastik & $\begin{array}{l}\text { nur dann nachgewiesenermaßen } \\
\text { sinnvoll, wenn Patient sich nicht } \\
\text { alleine mobilisieren kann }\end{array}$ & Zeitaufwand \\
\hline Akupunktur & $\begin{array}{l}\text { sehr selten Nebenwirkungen } \\
\text { keine Medikamenteneinnahme }\end{array}$ & $\begin{array}{l}\text { Effektivität nicht belegt, Kosten für } \\
\text { Patient, da Übernahme durch } \\
\text { Krankenkasse nicht immer } \\
\text { gewährleistet }\end{array}$ \\
\hline $\begin{array}{l}\text { lokale Injektions- } \\
\text { behandlung }\end{array}$ & keine Medikamenteneinnahme & $\begin{array}{l}\text { Effektivität nicht belegt, selten } \\
\text { Nebenwirkungen: Hautreaktionen, } \\
\text { Entzündungen, Durchführung } \\
\text { schmerzhaft }\end{array}$ \\
\hline
\end{tabular}

Gedanken, Befürchtungen, Erwartungen

Zum Teil wird der Patient schon aktiv seine Befürchtungen während der Vorstellung der Therapieoptionen äußern, darüber hinaus sollte er konkret sowohl nach seinen eigenen Vorstellungen der Behandlung, als auch nach Erwartungen an die Behandlung und Befürchtungen bezüglich der Therapien gefragt werden. Einige Patienten werden Befürchtungen zur Einnahme von Schmerzmitteln haben, andere werden befürchten, ohne eine krankengymnastische Behandlung oder ohne Röntgendiagnostik nicht ausreichend behandelt zu sein. Befürchtungen, die aktiv erfragt werden, können mit dem Patienten thematisiert und besprochen werden. Befürchtungen und Bedenken, mit denen der Patient alleine bleibt, können z. B. bei Bedenken zur Schmerzmedikation zur Nichteinnahme führen, die dem Arzt jedoch nicht mitgeteilt wird.

\section{Aushandeln, entscheiden, vereinbaren}

Die Therapieoptionen sollten mit dem Patienten unter dem Aspekt diskutiert werden, wie er sich am besten vorstellen kann, rasch wieder zur gewohnten Aktivität zurückzukehren. Dabei können Art und Stärke der Schmerzmedikation mit dem Patienten, der vielleicht bestimmten Medikamenten bereits kennt oder eingenommen hat, gemeinsam abgesprochen werden. Wenn ein Patient dabei große Bedenken vor einer Medikamenteneinnahme hat, kann 
versucht werden, dass Therapieziel ohne Medikamente zu erreichen, oder dem Patient ein Reserverezept für den Fall, dass er die Schmerzen doch behandeln möchte, mitgegeben werden. Auch wenn ein Patient eine nicht nachgewiesenermaßen wirksame Therapie wünscht kann mit ihm ein Therapieversuch vereinbart werden und kurzfristig überprüft werden, ob damit die Therapieziele erreicht werden konnten.

Genauso sollte mit dem Patienten gemeinsam entschieden werden, ob eine Krankschreibung Sinn macht. Dazu muss der Arzt vom Patienten genau erfragen, wie die Tätigkeit des Patienten genau aussieht und ob in der jetzigen Erkrankungssituation Möglichkeiten einer Anpassung der Tätigkeit gegeben sind. Darüber hinaus sollte er in Erfahrung bringen, ob es Konflikte am Arbeitplatz gibt und ob die Arbeitssituation als psychisch belastend erlebt wird. Eine Krankschreibung kann Sinn machen, wenn eine Arbeit sowohl körperlich sehr belastend und daher mit Beschwerden nicht ausführbar ist, als auch bei einer rein sitzenden Tätigkeit, um den Patienten zu ermöglichen sich mehr zu bewegen. Ebenso kann es bei psychisch belastenden Situationen am Arbeitsplatz sinnvoll sein, eine Entlastung durch eine kurzfristige Krankschreibung zu erwirken- und z. B. in dieser Zeit Lösungsstrategien für die belastende Arbeitssituation zu entwickeln.

Mit dem Patienten gemeinsam kann möglichst konkret geplant werden, wie Bewegung und Aktivität im Alltag aussehen können. Dabei können die Präferenz für bestimmte Sportarten, die zeitlichen und finanziellen Möglichkeiten des Patienten, die familiäre Situation sowie die infrastrukturellen und klimatischen Bedingungen berücksichtigt werden. Im Gespräch über die Bedingungen des Alltags des Patienten können dabei auch bestehende Belastungen im Privatleben angesprochen werden.

Da der Patient starke Bedenken bezüglich einer Medikamenteneinnahme hat, einigen sich Arzt und Patient darauf, dass der Patient zunächst alternativ mit physikalischen Maßnahmen (Wärme) versucht, die Beschwerden soweit zu lindern, dass er sich weitgehend normal bewegen kann. Da die Arbeit körperlich, durch einen Konflikt mit dem Vorarbeiter aktuell aber auch psychisch belastend ist, wird er für wenige Tage krankgeschrieben und wird in der Zeit Schwimmen und Radfahren gehen. Außerdem nimmt er sich vor, ein Gespräch mit dem Chef zu suchen um den aktuellen Konflikt am Arbeitsplatz zu lösen.

\section{Erfolge überprüfen, im Kontakt bleiben, Folgeentscheidungen}

Aus medizinischer Sicht ist es nicht notwendig, den Patienten bei zügiger und vollständiger Beschwerdebesserung wiedereinzubestellen. Dennoch sollte mit dem Patienten klar vereinbart werden, dass er sich bei Verschlechterung oder fehlender Besserung nach einem vereinbarten Zeitintervall wieder vorstellt. Um einer Chronifizierung vorzubeugen, ist eine Erweiterung der Therapie bei ausbleibender Besserung nach 4-6 Wochen notwendig und eine aktive Mitar- 
beit des Patienten wichtig. Analog zu den bereits dargestellten Schritten kann auch in dieser Situation wieder eine gemeinsam verantwortete Entscheidung getroffen werden. Insbesondere Maßnahmen wie eine Erweiterung der Medikation, Physiotherapie, Bildgebung oder bei bestehenden psychischen Belastungen eine psychotherapeutische Unterstützung können dabei mit ihren Vor- und Nachteilen aus medizinischer Perspektive und aus Patientensicht diskutiert werden. Wenn bereits initial eine gemeinsame Behandlungsplanung erfolgt, wird der Patient sich auch im Weiteren mitverantwortlich an der Therapieentscheidung und Therapie beteiligen, was essenziell für die Therapiemaßnahmen zur Verhinderung oder Behandlung von chronischen Rückenschmerzen ist. Für die in der weiteren Entwicklung sehr selten anstehende Entscheidung zu einer operativen Maßnahme ist nachgewiesen, dass eine Einbeziehung von Patienten mit Bandscheibenerkrankungen zu niedrigeren Operationsraten bei gleichem Outcome für Beschwerdebesserung und Funktionalität führt.

\subsection{Shared Decision-Making - Hilfe bei Kommunikation und Konsultation im Alltag}

Wie anhand eines skizzierten Fallbeispiels dargelegt, bietet das Konzept des Shared decision Making ein klar strukturiertes Vorgehen um eine gelungene Kommunikation und eine verbesserte Einbeziehung des Patienten zu erreichen.

Ärzte, die nach einer Ärzteschulung im klinischen Alltag eine gemeinsame Entscheidungsfindung im Shared Decision-Making umsetzen, sind oft zunächst unsicher und skeptisch, wie sich die Theorie und die daraus hergeleiteten Handlungsschritte in der Praxis umsetzen lassen: Ob die Patienten überhaupt mitentscheiden wollen? Wie zeitaufwändig erweist sich eine solche Kommunikation? Erreicht man damit überhaupt etwas? Nach den ersten Erfahrungen im Praxisalltag berichten sie häufig, dass Patienten nicht, wie befürchtet verunsichert reagieren, sondern, nach manchmal anfänglicher Überraschung, die Einbeziehung gerne annehmen und zufrieden wirkend die Sprechstunde verlassen. Die Ärzte haben dabei auch den Eindruck, dass Patienten in ihrer Behandlung aktiver werden und die Therapie mir mehr eigener Verantwortung und Engagement durchführen. Nach anfänglichem zeitlichen Mehraufwand wird mit zunehmender Übung und Routine auch insgesamt kein bedeutsamer Zeitverlust mehr wahrgenommen: Oft berichten Ärzte, dass zwar Erstgespräche zu Therapieentscheidungen etwas länger benötigen, bei Folgekonsultationen und im weiteren $\mathrm{Pa}$ tientenkontakt aber durch den engagierten Patienten wieder Zeit „eingespart" wird. 


\section{Bringt das wirklich etwas?}

Dieses subjektive Erleben und diese einzelnen Arztberichten stimmen gut mit den Erkenntnissen aus Übersichtsarbeiten zu Effekten des Shared DecisionMaking überein: Die meisten Patienten wünschen eine Einbeziehung in medizinische Entscheidungen (Coulter 2003) und eine Einbeziehung des Patienten führt zu besserem Wissen des Patienten über die Erkrankung, realistischeren Erwartungen an die Therapie, einer aktivere Teilnahme am medizinischen Entscheidungsprozess und größerer Patientenzufriedenheit (Loh, Simon et al. 2007, Scheibler et al. 2003). Dabei kommt es weder zur Verunsicherung und erhöhten Angst der Patienten noch - unter den Vorraussetzungen, dass Ärzte eine entsprechende Schulung erhalten haben und Patienteninformationen zu ihrer Unterstützung einsetzen können - zu einer Verlängerung der Konsultationszeit (Loh, Leonhart et al. 2007).

\section{Umsetzung und praktische Hilfen}

Die praktische Umsetzung des Shared Decision-Making ist erlern- und trainierbar (Bieber 2007), Ärzte die entsprechend geschult werden, erreichen bereits nach kurzer Zeit und mit zeitlich begrenztem Fortbildungsaufwand (ca. 2 Halbtagstermine) eine deutlich verbesserte Einbeziehung der Patienten und größere Behandlungszufriedenheit. Leider finden solche Schulungen, in denen die Handlungsschritte der gemeinsamen Entscheidungsfindung auch praktisch in Rollenspielen vermittelt werden, momentan noch lediglich im Rahmen von Interventionsstudien regelmäßig statt. Der interessierte Arzt ist im Moment daher auf Internetressourcen zu verweisen: Unter www.patientals-partner. de sind ein cme-Fortbildungsmodul, Trainingsmaterialien, Literaturempfehlungen und weiterführende Informationen zu finden.

Zur praktischen Unterstützung bei der Kommunikation sind schriftliche Patienteninformationen hilfreich, die mitgegeben werden können und in denen der Patient nochmals Hintergrundwissen und Therapieoptionen nachlesen kann. Als deutschsprachige Quellen sind hier die DEGAM- Patienteninformation Kreuzschmerzen und die Patientenleitlinien der Universität Witten-Herdecke (www.patientenleitlinien.de) empfehlenswert.

\subsection{Fazit}

Für eine leitlinien- und patientengerechte Behandlung von Kreuzschmerzen kann zur Erleichterung der Kommunikation und Verbesserung des Behandlungsergebnisses auf die praktischen Handlungsschritte und Kommunikationsstrategien einer gemeinsamen Entscheidung zurückgegriffen werden. Eine gemeinsam mit dem Patienten getroffene Entscheidung führt zu einer größeren Zufriedenheit des Patienten und dazu, dass er sich mitverantwortlich an der Therapie beteiligt. 


\section{Literatur}

Becker A, Niebling W, Chenot JF et al.: DEGAM Leitlinie Kreuzschmerzen. Düsseldorf 2003

Becker A, Kögel K, Donner-Banzhoff N et al.: Kreuzschmerzpatienten in der hausärztlichen Praxis: Beschwerden, Behandlungserwartungen und Versorgungsdaten. Zeitschrift für Allgemeinmedizin 2003; 79: 125-131

Bieber C, Mueller KG, Blumenstiel K, Hochlehnert A, Wilke S, Eich W: A Shared Decision Making communication training programme for physicians treating fibromyalgia patients - effects of a randomized controlled trial. | Psychosom Res 2007; 57(2): 61-69

Biering-Sorensen F, Thomsen C: Medical, social and occupational history as risk indicators for back trouble in the general population. Spine 1986; 1: 720-725

Coulter A, Magee H: The European patient of the future. Berkshire: Open University Press 2003.

Deyo RA, Cherkin DC, Weinstein / et al.: involving Patients in clinical decisions. Impact of an interactive Video Program on Use of Back Surgery. Medical Care 2000; 38/9: 959-969

Deyo RA, Diehl AK: Psychosocial predictors of disability in patients with low back pain. Journal of Rheumatology 1988; 15(10): 1557-64

Deyo RA, Weinstein DO: Low back pain. New England Journal of medicine 2001; 344: 363-370

Gatchel RI, Polatin PB, Mayer TG: The dominant role of psychosocial risk factors in the development of chronic low pain disability. Spine 1995; 20: 27002-2709

Göbel H: Epidemiologie und Kosten chronischer Schmerzen. Spezifische und unspezifische Rückenschmerzen. Schmerz 2001; 15: 92-98

Lehmann TR, Spratt KF, Lehmann KK: Predicting long-term disability in low back injured workers presenting to a spine consultant. Spine 1993; 18: 1103-1112

Loh A, Leonhart R, Wills C, Simon D, Härter M: The impact of patient participation on adherence and clinical outcome in primary care of depression. Patient Educ Couns 2007; 65: 69-78

Loh A, Simon D, Kriston L, Härter M: Patientenbeteiligung bei medizinischen Entscheidungen. Effekte der partizipativen Entscheidungsfindung aus systematischen Reviews. Dtsch Arztebl 2007; 104(21): A 1483-8

Nelson CW: Helping patients decide: from Hippocrates to videodiscs - An application for patients with low back pain. Journal of Medical Systems 1988; 12/1: 1-9

Scheibler F, Janssen C, Pfaff $\mathrm{H}$ : Shared Decision Making: Ein Überblicksartikel über die internationale Forschungsliteratur. Soz Praventivmed 2003; 48: 11-23

Schiottz-Christensen B, Nielsen GL, Kjaer V et al.: Long-term prognosis of acute low back pain in patients seen in general practice: a 1 year prospective follow-up study. Family practice 1999; 16: 223-232

Schmidt CO, Raspe H, Pfingsten $\mathrm{M}$ et al.: Back pain in the german adult population. Prevalence, severity, and sociodemographic correlates in a multiregional survey. Spine 2007; 32: 2005-2011.

Simon D, Loh A, Härter M: Shared Decision Making bei medizinischen Entscheidungen: neue Wege für Ärzte und Patienten in der Orthopädie. Orthopädische Praxis 2008; 44/3: 137-142 